\title{
Thrombophilia, Pulmonary Embolism (PE) and Deep Venous Thrombosis (DVT) in pregnant women: a brief communication
}

\section{Abstract}

Venous thromboembolism (VTE), deep vein thrombosis and pulmonary embolism, are among the main causes of morbidity and mortality in pregnancy with an estimated incidence of 1 per thousand women years. Despite identification of maternal and pregnancy-specific risk factors for development of pregnancy-associated venous thromboembolism, limited data are available to inform on optimal approaches for prevention. The relatively low overall prevalence of pregnancyassociated venous thromboembolism has prompted debate about the validity of recommendations, which are mainly based on expert opinion, and have resulted in an increased use of pharmacological thromboprophylaxis in pregnancy and postpartum.

Keywords

Venous thromboembolism, pregnancy, thromboprophylaxis

Pulmonary embolism (PE) and deep venous thrombosis (DVT) are two components of a single pathophysiological process called venous thromboembolism (VTE). The PE usually occurs as a result of DVT, but often thrombosis is not diagnosed before the occurrence of embolia [1].

Thrombophilia are common disorders that increase the risk of pregnancy-associated venous thromboembolism and pregnancy loss and can also increase the risk of placenta-mediated pregnancy complications (severe pre-eclampsia, small-for-gestational-age infants, and placental abruption). Thrombophilia are common acquired or genetic predispositions to develop venous thromboembolism. Venous thromboembolism, which comprises deep vein thrombosis and pulmonary embolism, is more frequent in pregnant women than in non-pregnant age-matched women [2].
Camila Grangeiro Fernandes ${ }^{1}$, André Carvalho Schlachter ${ }^{1}$, Naiala de Souza Ribeiro Sales', Antonio Gilvan Teixeira Júnior ${ }^{1}$, Modesto leite Rolim Neto'.

1 Faculty of Medicine. Federal University of Cariri. Barbalha, Ceara, Brazil. 
Placenta-mediated pregnancy complications, comprising pre-eclampsia, birth of a small-for-gestational-age infant, placental abruption, or pregnancy loss, are also common and lead to substantial maternal and fetal or neonatal morbidity and mortality. Patients with previous placenta-mediated pregnancy complications have a raised risk of both recurrent placenta-mediated pregnancy complications and venous thromboembolism during subsequent pregnancies [2].

Thromboembolic events are among the leading causes of maternal morbidity and mortality in pregnancy and postpartum period. While the main cause of maternal death in developing countries is bleeding, thromboembolic events are the leading cause of maternal death in developed countries, where death from bleeding is prevented. The incidence of these events varies between 0.76 to 1.72 per 1,000 pregnancies, four to five times more often than women not pregnant [1].

Thrombotic risk factors have been associated with pregnancy complication. Many previous studies have found an association between inherited thrombophilia and adverse pregnancy outcomes, however there has been wide variability in the strength of the association and some studies have not found a relationship. Despite significant racial disparities in rates of adverse pregnancy outcomes, most studies examining the relationship of thrombotic risk factors in women with adverse pregnancy outcomes have been performed in white populations, and have focused on Factor $\vee$ Leiden and prothrombin gene mutations, thrombotic risk factors known to be less prevalent among black populations [3].

The prevalence of diagnosed thrombophilia differed by race. Overall, thrombophilia was more common in white women with adverse pregnancy outcomes compared to black women (44\% vs $30.3 \%, p=0.04)$, although this difference was primarily due to the difference in factor $\mathrm{V}$ Leiden mutation (19\% vs 3\%, p b 0.01). However, pro- tein S deficiency was significantly more common in black women (15.2\% vs $5.8 \%, p=0.02)$. When assessed among the subgroup of women who were not pregnant at study enrollment (252 white and 32 black), protein $\mathrm{S}$ deficiency was still more common in black women but the difference did not reach statistical significance $(13.5 \%$ vs $5.5 \%, p=0.08)$. None of the women with protein $S$ deficiency had sickle cell disease, renal disease, or HIV. Antithrombin deficiency was also more common among black women (6\% vs $1.5 \%, p=0.04)$. There were no racial differences in the frequency of laboratory testing for protein $\mathrm{S}$ or antithrombin [3].

A meta-analysis showed that two-thirds of cases of DVT occurred during pregnancy and were equally distributed in the three quarters, while $43-60 \%$ of episodes of PE occurred in the postpartum period. DVT in the lower limb is the most common type of venous thromboembolism during pregnancy. Approximately $30 \%$ of the isolated cases are episodes of pulmonary embolism associated with DVT silent, and in patients presenting the symptoms of DVT and pulmonary embolism the frequency of $P E$ is around $40-50 \%$. The risk of venous thromboembolism, added to pregnancy status, and even more if it is combined with other factors such as family or personal history of VTE, thrombophilia, age over 35, obesity and high parity. Cesarean section also significantly increases the risk of thromboembolic events compared to vaginal delivery [1].

Thrombophilia, an entity that includes hereditary disorders and acquired associated with an increased tendency to develop VTE is a major determinant of this complication during pregnancy. The main hereditary conditions of thrombophilia include: antithrombin deficiency of $\mathrm{C}$ and $\mathrm{S}$ proteins; mutations on prothrombin gene and factor $V$ Leiden; homozygous to methylenetetrahydrofolate reductase and hyperhomocysteinemia. As acquired thrombophilic states, we can mention the antiphospholipid syndrome (APS) and the nephrotic syndrome with consequent decreased levels of antithrombin [1]. 
A personal history of VTE is a significant risk factor for VTE during pregnancy. Women with a previous VTE have a 3 to 4 fold increased risk of recurrent events during subsequent pregnancies, with studies demonstrating rates of recurrence of $6 \%$ to $8 \%$ during the antepartum and postpartum period, respectively. In a prospective study of 125 pregnant women with a previous single episode of VTE, the incidence of antepartum and postpartum recurrence without prophylaxis was $2.4 \%(95 \%$ confidence interval [Cl] $0.2 \%-6.9 \%$ ) and $2.5 \%$ (95\% Cl 0.5\%-7.0\%), respectively. Evidence regarding other prognostic factors for recurrent VTE has been conflicting. One post hoc analysis found a low risk of recurrence in women without thrombophilia with a temporary risk factor, including oral contraceptives or pregnancy-related VTE, at the time of their first event. Alternatively, retrospective studies have failed to show a consistent association between temporary risk factors and recurrent VTE. Investigators found women with a first VTE provoked by use of oral contraceptives or related to pregnancy or the postpartum period were at a higher risk of recurrent thromboembolism than those who experienced an episode of VTE provoked by a no hormonal transient risk factor; it should be noted however that this difference did not reach statistical significance. For women with a history of prior VTE, the American College of Chest Physicians classify risk as low, moderate, or high for recurrent VTE during pregnancy. Low-risk patients include those who have had a single episode of VTE associated with a transient risk factor, not including pregnancy or use of estrogen; moderate to high-risk patients include those who experienced an idiopathic VTE, pregnancy or estrogen-related VTE, or multiple prior VTE not currently receiving anticoagulation [4].

Worldwide, numerous women achieve pregnancy by the aid of in vitro fertilization (IVF). Until recently, IVF treatment was regarded a risk factor for thrombosis based on reviews of case reports only.
During IVF, controlled ovarian stimulation leads to multiple oocytes and supra-physiological levels of estrogens [5].

The consequence is a pro-coagulant like state during pregnancy. Pregnancy per se increases the risk of venous thromboembolism (VTE), especially during the third trimester and the first 6 weeks after delivery. VTE, especially pulmonary embolism, remains a leading cause of maternal mortality [5].

Four studies have provided important evidence on the issue of whether IVF is a risk factor for VTE. Jacobsen et al. found that the VTE risk during pregnancy and the post-partum period was significantly increased compared with other pregnancies, and Henriksson et al. and Rova et al. showed a significantly increased VTE risk among IVF-pregnancies during the first trimester. A group recently reported that there was no evidence of increased VTE risk after IVF treatment in unsuccessful cycles [5].

Current smokers had no apparent increased risk of venous thromboembolism relative to non-smokers, with a rate ratio of $0.9(95 \% \mathrm{Cl}, 0.7$ to 1.2$)$. Women whose information on smoking was missing had a rate ratio of venous thromboembolism of 1.8 (95\% Cl 1.2-2.7) compared with non-smokers. Hyperemesis was associated with an increased risk of venous thromboembolism in pregnancy, with a rate ratio of $2.5\left(95 \% \mathrm{Cl}_{1} 1.4\right.$ to 4.5$)$. Women with a BMI between 25 and 29.9 had a rate ratio of venous thromboembolism of $1.4(95 \% \mathrm{Cl}, 1.0$ to 2.0$)$, relative to women with a BMI of 18.5 to 24.9 , whereas values of BMI greater than 25 were not associated with notably increased risk for venous thromboembolism. Multiple pregnancies were associated with a rate ratio for venous thromboembolism of 2.8 (95\% $\mathrm{Cl}, 1.9$ to 4.2 ) compared with singleton pregnancies. Receiving antibiotic treatment for infection during pregnancy was associated with a rate ratio of venous thromboembolism of $1.8(95 \% \mathrm{Cl}, 1.5$ to 2.3$)$. For women who had a discharge diagnosis of infection during pregnancy the rate ratio was 4.3 (95\% $\mathrm{Cl}, 2.7$ to 7.1 ) adjusted for all confounders. Hospi- 
talization 3-7 days during pregnancy increased the risk for venous thromboembolism 12.2 fold (95\% $\mathrm{Cl}$, 8.7-17.0). Shorter and longer stays also increased the risk for venous thromboembolism, although less so. The risk associated with polyhydramnios was not assessed as there were few women with a diagnosis of polyhydramnios and only one case of venous thromboembolism among this women [6].

Smoking at the beginning of pregnancy was only weakly associated with risk of venous thromboembolism during the puerperal period. Treatment with antibiotics during pregnancy was associated with a rate ratio of venous thromboembolism in the puerperal period of $1.3(95 \% \mathrm{Cl}, 1.0$ to 1.6$)$; the corresponding figure for women who were treated during the puerperal period was 1.4 (95\% $\mathrm{Cl}, 1.0$ to 1.9). Women with a hospital diagnosis of infection in pregnancy or the puerperal period had a rate ratio of venous thromboembolism of 2.4 (95\% Cl, 1.5 to 3.8$)$ and $5.0(95 \% \mathrm{Cl}, 2.4$ to 10.6$)$, respectively [6].

There was only a weak association between risk of venous thromboembolism and being hospitalized less than a week in the puerperal period, whereas women with a hospitalization of 8-14 days or more than 14 days had a rate ratio of $4.2(95 \% \mathrm{Cl}, 3.0$ to 6.0$)$ and $5.9\left(95 \% \mathrm{Cl}_{1} 4.0\right.$ to 8.8$)$, respectively. Although there was a strong association between a multiple pregnancy and risk of venous thromboembolism during pregnancy, the rate ratio in the puerperal period after carrying more than one fetus was only $1.3(95 \% \mathrm{Cl}, 0.6$ to 2.6$)$ [6].

Compared with women of normal BMI, women with BMI between 25 and 29.9, 30 and 34.9 and $35+$ had a rate ratio of puerperal venous thromboembolism of $1.7(95 \% \mathrm{Cl}, 1.1$ to 2.7$), 2.1(95 \% \mathrm{Cl}, 1.1$ to 3.9$)$, and $3.5(95 \% \mathrm{Cl}, 1.8$ to 6.7$)$, respectively. Women undergoing elective caesarean sections or emergency caesarean sections had a rate ratio of puerperal venous thromboembolism of $2.1(95 \% \mathrm{Cl}$, 1.4 to 3.1$)$ and $3.0\left(95 \% \mathrm{Cl}_{2} 2.3\right.$ to 4.0$)$, respectively, compared with women giving birth vaginally.
Women diagnosed with major postpartum bleeding had a rate ratio of puerperal venous thromboembolism of $1.4(95 \% \mathrm{Cl}, 1.0$ to 2.1) [6]. We estimated the risk of puerperal venous thromboembolism for women hospitalized more than 1 day during pregnancy according to selected causes of admission to hospital [6].

Admission for preeclampsia and a bleeding episode including abruption placenta and placenta previa during pregnancy had a strong thrombogenic effect that persisted in the puerperal period. The rate ratio for preeclampsia was $5.0(95 \% \mathrm{Cl}$, 3.1 to 7.8$)$ and a bleeding episode $2.1(95 \% \mathrm{Cl}, 1.1$ to 4.3). We found only a modestly elevated increased risk of puerperal thromboembolism when the primary cause of admission during pregnancy was threatened preterm labor (1.6 (0.7-4.0)), whereas when this diagnosis was combined with one or more other diagnoses it was considerably increased (3.4 (2.0-6.0)) [6].

Extensive clinical experience and retrospective studies have established heparin as the anticoagulant for safe use during pregnancy, do not cross the placenta, and therefore does not cause bleeding or teratogenic effect on the unborn child [1].

Some authors recommend warfarin use during pregnancy in some special circumstances, such as in women with mechanical heart valves, those who had recurrence while receiving heparin and those with contraindications to the use of heparin [1].

The anticoagulants warfarin does not induce effects in children fed breast milk and therefore can be used safely in the post-period childbirth [1].

Low-molecular-weight heparin (LMWH) is the recommended drug for antithrombotic prophylaxis and therapy of VTE, and its use in pregnancy has greatly increased in recent years. However, maternal mortality has not decreased, as reported by the World Health Organization and in a recent worldwide report. The suggested dose varies according to LMWH molecules, being $40 \mathrm{mg}$ for sodium enoxaparin and 2,850 international unit for calcium na- 
droparin, once a day subcutaneously, that can be increased according to maternal increase of body weight. The choice of type and dose of LMWH is based on physicians' experiences and varies among hospitals and countries [7].

The goal of thromboprophylaxis is to provide VTE protection with minimal side effects for the mother and no effects on the fetus. Although today we have a huge choice of anticoagulant and antiplatelet agents, heparin is still the anticoagulant of choice for VTE prophylaxis and treatment in pregnancy. Currently, LMWHs have replaced UFH as the first choice anticoagulant. LMWHs are at least as effective as UFH, but produce more predictable anticoagulant response due to better bioavailability (90-100\% after subcutaneous administration), longer half-life (4-6 h), dose-independent renal clearance, decreased affinity for heparin-binding proteins, endothelial cells and macrophages. Effective anticoagulation can be achieved by subcutaneous application of LMWH once daily, with no need for routine laboratory monitoring of anti-factor Xa activity in plasma. Nevertheless, in certain situations LMWH dose adjustment is necessary and that demands anti-Xa level assessment [8].

\section{References}

1. Pontes, DM; Pimentel, LGB Eventos tromboembólicos na gestação e puerpério:revisão sistemática e recomendação atual. Femina; 2013, 41(1), jan-fev.

2. Marc A Rodger, MD et al. Antepartum dalteparin versus no antepartum dalteparin for the prevention of pregnancy complications in pregnant women with thrombophilia (TIPPS): a multinational open-label randomised trial. The Lancet, 2014, 384,(8-14): 1673-1683.

3. Philipp, C. S. et al. Differences in Thrombotic Risk Factors in Black and White Women with Adverse Pregnancy Outcome. Thromb Res. 2014; 133(1): 108-11.

4. Armstrong, E.M.; Bellone, J.M.; Hornsby, L.B. Pregnancy-Related Venous Thromboembolism. J Pharm Pract. 2014, 17; 27(3): 243252.

5. Hansen AT, Kesmodel US, Juul S, Hvas AM. Increased venous thrombosis incidence in pregnancies after in vitro fertilization. Hum Reprod. 2014, 29(3): 611-7.
6. R. A.,Virkus et al.Risk Factors for Venous Thromboembolism in 1.3 Million Pregnancies: A Nationwide Prospective Cohort. PLoS One. 2014; 9(5): e96495.

7. Testa S. et al.The "Pregnancy Health-care Program" for the prevention of venous thromboembolism in pregnancy. Intern Emerg Med. 2014, 31.

8. Kutlesić, M.; Kutlesić, R.; Koraćević, G. Significance, aetiology and prevention of venous thromboembolism in pregnancy and puerperium. Vojnosanit Pregl. 2014, 71(6): 580-7.

\section{Comment on this article:}
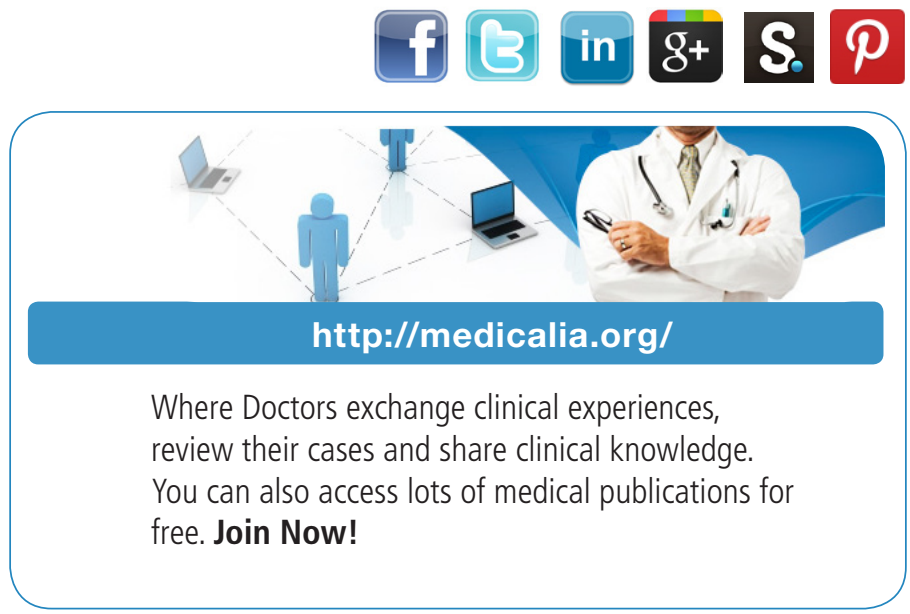

Publish with iMedPub

http://www.imed.pub

International Archives of Medicine is an open access journal publishing articles encompassing all aspects of medical science and clinical practice. IAM is considered a megajournal with independent sections on all areas of medicine. IAM is a really international journal with authors and board members from all around the world. The journal is widely indexed and classified Q1 in category Medicine. 